\title{
FATORES PREDITIVOS DO TRATAMENTO OPERATÓRIO NA ÚLCERA PÉPTICA HEMORŔGGICA
}

\author{
José Gustavo Parreira*, tulio Eduardo flesch Pfiffer, Silvia Soldá, Carlos Malheiros, Samir Rasslan \\ Trabalho do Serviço de Emergência e do Departamento de Cirurgia da Faculdade \\ de Ciências Médicas da Santa Casa de São Paulo, SP
}

RESUMO - OBjetivo. Avaliar os fatores preditivos de tratamento operatório nos doentes com úlceras pépticas hemorrágicas.

Métodos. Através de um estudo retrospectivo, foram analisados os prontuários dos doentes com úlceras pépticas hemorrágicas admitidos de 1998 a 2001 . Foram avaliados 200 doentes, com idade entre 17 e 97 anos e média etária de $52 \pm 18$ anos, sendo 153 (76,5\%) do sexo masculino. Dados vitais à admissão, antecedentes mórbidos, achados e tratamento endoscópico, bem como a evolução e tratamento definitivo foram revisados. Empregou-se 0 teste $t$ de student e qui-quadrado para a análise estatística, considerando-se 0 valor de $p<0,05$ como significativo.

Resultados. Vinte e sete (I3,5\%) doentes foram admitidos em choque. À endoscopia da admissão, I0I úlceras localizavam-se no duodeno e 99 no estômago, sendo classificadas como Forrest la em cinco (2,5\%), lb em 20 (10\%), lla em 48 (24\%) e llb em 48 (24\%).
Vinte e cinco doentes (12,5\%) foram operados, 23 (92\%) sendo submetidos à gastrectomia parcial com reconstrução a Billroth II. Os fatores associados à necessidade de tratamento operatório foram os antecedentes de alcoolismo $(p=0,002)$, tabagismo $(p=0,02)$, diabetes mellitus $(p=0,01)$ e doença péptica $(p=0,05)$, além de choque à admissão $(p<0,00 \mathrm{I})$ e lesões Forrest la à endoscopia. Os doentes com lesôes Forrest llb que não receberam tratamento endoscópico à admissão necessitaram tratamento cirúrgico com maior freqüência $(p=0,012)$.

Conclusão. Alcoolismo, tabagismo, antecedentes de doença péptica ou diabetes mellitus, instabilidade hemodinâmica à admissão e lesões Forrest la são fatores preditivos de tratamento operatório nos doentes com úlceras pépticas hemorrágicas.

UNITERMOS:Úlcerapéptica. Úlcera péptica hemorrágica-terapia/cirurgia.

\section{INTRODUÇÃO}

Admite-se que até $45 \%$ das hemorragias digestivas altas são causadas por úlceras pépticas'. Foram descritos vários fatores de risco para esta complicação, como a administração de drogas antiinflamatórias não esteróides, anticoagulantes orais, corticoesteróides, bem como a presença de Helicobacter pylorii e idade avançada ${ }^{2-4}$.

Atualmente, a endoscopia digestiva altaéo procedimento de escolha nos doentes estáveis hemodinamicamente, com finalidade diagnóstica e terapêutica. Métodos endoscópicos para o controle do sangramento alcançam o sucesso em $83 \%$ a $93 \%$, reservando a laparotomia para casos selecionados ${ }^{5-8}$. Diante da falha da hemostasia endoscópica, caracterizada pela recidiva hemorrágica, deve-se indicar o tratamento operatório. Outras variáveis também são analisadas para a indicação precisa do

*Correspondência:

Rua Dona Veridiana 167, apartamento 83

Santa Cecilia, São Paulo-SP-CEP 01238-010

Telefone:(11) 2213458-2240122-ramal 5082 igparreira@uol.com.br tratamento operatório, como idade, local e características endoscópicas das úlceras, história prévia de utilização de fármacos antiinflamatórios não esteróides, doença péptica crônica, tipo sangüíneo evolume de concentrados de hemácias transfundido ${ }^{9}$. Esta variedade de fatores muitas vezes torna difícil a decisão quanto à melhor forma de tratamento.

Contudo, sabe-se que a instabilidade hemodinâmica e anemia são fatores relacionados a complicações e maior letalidade nos doentes operados por úlcera péptica hemorrágica ${ }^{10-12}$. Portanto, postergar a indicação de uma operação pode determinar pior prognóstico.

Atualmente buscam-se fatores que auxiliem na indicação precoce da operação ${ }^{13}$. São descritas variáveis associadas à maior probabilidade de falha no tratamento endoscópico, e estas incluem as úlceras de diâmetro superior a $2 \mathrm{~cm}$, coto vascular visível maior que I $\mathrm{mm}$, e as úlceras de parede posterior de duodeno ou altas em pequena curvatura gástrica $^{14}$. Forrest et al., em 1974, descreveram uma classificação para as úlceras pépticas hemorrágicas, também com o objetivo de orientar o tratamento e avaliar prognóstico $(\text { Tabela I) })^{15}$. Desta forma, é possível identificar os doentes com maior probabilidade de necessitar tratamento operatório.

Oobjetivo deste estudo é analisar os fatores preditivos de tratamento operatório nos doentes admitidos com úlceras pépticas hemorrágicas.

\section{Métodos}

Realizou-se um estudo retrospectivo através da análise dos prontuários dos doentes admitidos por hemorragia digestiva alta no Serviço de Emergência do Departamento de Cirurgia da Santa Casa de São Paulo, no período de 1998 a 2001 . Foram avaliados 200 doentes, com idade de 17 a 97 anos, e média etária de $52 \pm 18$ anos. Cento e cinqüenta e três $(76,5 \%)$ eram do sexo masculino.

Incluíram-se no estudo aqueles submetidos à endoscopia digestiva alta, com diagnóstico de úlcera péptica hemorrágica e sangramento ativo ou recente. Foram excluídos os portadores de doenças hepáticas e hipertensão portal, bem como aqueles que apresentavam doenças hematológicas, distúrbios de coagulação e com cirurgia gastroduodenal pré- 
Tabela I - Classificação de Forrest para as úlceras pépticas hemorrágicas

\begin{tabular}{cc}
\hline Forrest & Descrição endoscópica \\
Ia & Sangramento emjato provenientedalesão \\
Ib & Sangramentodifusoprovenientedalesão \\
Ila & Presençade cotovascular \\
IIb & Presençade cóaguloaderido ao fundo daúlcera \\
IIc & Presençade pontos dehematina fibrina \\
III & Ausênciadesinais de sangramento \\
\hline
\end{tabular}

Tabela 2 - Classificação das lesões da amostra segundo Forrest, freqüência de tratamento e operação

\begin{tabular}{cccc}
\hline Classificação & Freqüência na amostra & Tratamentoendoscópico & Operados \\
Ia & $2,5 \%$ & $100 \%$ & $100 \% *$ \\
Ib & $10 \%$ & $95 \%$ & $15 \%$ \\
Ila & $24 \%$ & $95 \%$ & $14,7 \%$ \\
IIb & $24 \%$ & $66 \%$ & $12,5 \%$ \\
Ilc & $39 \%$ & $10 \%$ & $5 \% \%$ \\
\hline
\end{tabular}

$* p<0,001$

via. Considerou-se o choque como a pressão arterial sistólica abaixo de $90 \mathrm{~mm} \mathrm{Hg}$, acompanhada de frequiência cardíaca acima de 100 batimentos por minuto. Nos doentes com mais de uma lesão ulcerada, considerou-se para análise apenas a que apresentasse sinais de sangramento à endoscopia.

O protocolo de atendimento incluiu acesso venoso e infusão de cristalóides até a recuperação da estabilidade hemodinâmica, seguida da avaliação endoscópica. O jejum foi mantido por 24 horas após o procedimento, associado à reposição hídrica e eletrolítica. Ranitidina endovenosa foi administrada em doses de $150 \mathrm{mg}$ a $600 \mathrm{mg}$ ao dia. O diagnóstico de ressangramento foi baseado em diminuição progressiva do hematócrito, acompanhada de taquicardia e/ou hipotensão arterial.

Foram analisadas: idade, doenças associadas, drogas utilizadas, transfusões sanguíneas, características endoscópicas e classificação de Forrest da úlcera péptica, bem como a evolução durante a internação. Foram também avaliadas as formas de tratamento endoscópico e operatório e suas complicações.

Para a determinação dos fatores preditivos de tratamento operatório, foram comparadas as variáveis entre os doentes submetidos ou não à operação. Os testes qui-quadrado e t student foram empregados para a análise estatística, considerando $p<0,05$ como significativo.

\section{Resultados}

\section{Admissão}

Cento e dezessete doentes apresentavam hematêmese (58,5\%). Amédia edesviopadrão da pressão arterial sistólica à admissão foi $117 \pm 23 \mathrm{~mm} \mathrm{Hg}$. Vinte e sete (I3,5\%) doentes foram admitidos em choque. A hemoglobina sérica variou de 4 a $16 \mathrm{~g} / \mathrm{dL}$, com média de $9,9 \pm 2 \mathrm{~g} / \mathrm{dL}$, sendo que 88 (44\%) apresentavam valores menores que $10 \mathrm{~g} / \mathrm{dL}$.

Foram encontrados antecedentes de doença péptica em 80 casos (40\%), e, do total, $52(26 \%)$ haviam apresentado algum episódio prévio de hemorragia digestiva. Quarenta e quatro doentes $(22 \%)$ tinham antecedentes de hipertensão arterial sistêmica, 17 (8,5\%) de diabetes mellitus, 49 (24,5\%) de alcoolismo, $69(34,5 \%)$ de tabagismo. Quarenta e um (20,5\%) haviam ingerido antiinflamatórios não esteróides na semana anterior à admissão.

\section{Endoscopia digestiva alta}

Aendoscopia digestiva alta foi realizada em todos os doentes. A úlcera se encontrava no duodeno em $\mathrm{I} 0 \mathrm{I}$ casos $(50,5 \%)$ e no estômago em 99 (49,5\%). A média dos diâmetros das lesões foi de 9,8 $\pm 6,6$ milímetros. Cinco doentes $(2,5 \%)$ tinham lesões classificadas como Forrest la, 20 (10\%) como lb, 48 (24\%) como lla, 48 (24\%) como llb, e 79 (39,5\%) como llc (Tabela 2).

Otratamento endoscópico foi realizado em I 10 casos (55\%), sendo a injeção de adrenalina
I: 10000 , empregada em 90 doentes (81\%), a opção mais freqüente (Tabela 2). Foram também empregadas a cauterização com heater probe em 17 (15\%), esclerose com álcool em cinco (4,5\%) e métodos hemostáticos não especificados em três doentes.

\section{Evolução}

Transfusões de concentrados de hemácias foramnecessáriasem 108 casos (54\%), variando de 300 a 3300 mililitros. Seis doentes foram submetidosaumanova endoscopiadigestivaalta.

No total, 25 (I2,5\%) foram operados, sendo que dez (40\%) tinham idade superior a 60 anos, nove $(36 \%)$ foram admitidos em choque e 20 (80\%) com hemoglobina sérica menor que $10 \mathrm{~g} / \mathrm{dL}$. Treze apresentavam úlceras gástricase 12 úlceras duodenais. A gastrectomia parcial com reconstrução a Billroth II, pré-cólica, oralis totalis e anisoperistáltica foi realizada em 23 doentes (92\%). Três apresentaram complicações relacionadas ao procedimento operatório, representadas por duas fístulas do coto duodenal e uma deiscência de gastroenteroanastomose.

Seis doentes (3\%) faleceram, todos haviam sido operados e a causa do óbito foi sepse. Quatro destes apresentaram pneumonia com insuficiência respiratória e, outros dois, peritonite decorrente da deiscência de coto duodenal egastroenteroanastomose.

\section{Fatores associados à necessidade de tratamento operatório}

Os doentes com antecedentes de tabagismo, alcoolismo, doença péptica ou diabetes mellitus, bem como os admitidos em choque foram operados significativamente com maior freqüência (Tabela 3). Comparando-se os doentes operados e não operados, houve diferença significativa entre as médias de hemoglobina sérica, hematócrito, diâmetro da úlcera e necessidade de transfusões sanguínea (Tabela 4). A classificação de Forrest associouse significativamente com a necessidade de tratamento operatório $(p<0,00 \mathrm{I})$ (Tabela 2). Os doentes com lesões Forrest lb, lla e llb necessitaram de operação em $12,5 \%$ a I5\%, e os Forrest llc raramente foram operados.

Análise dos doentes Forrest lb, lla e Illb

Estegrupofoirepresentado por II6casos, 97 dos quais receberam tratamento endoscópico (83\%).Setenta(60\%)necessitaram transfusõesde concentrados de hemácias. Dezesseis (13\%) 


\begin{tabular}{lccc}
\hline \multicolumn{4}{c}{ Tabela 3 - Fatores associados à necessidade de tratamento operatório } \\
\hline Variável & Freqüência na amostra & Operados & $\mathbf{p}$ \\
Sexomasculino & $76 \%$ & $13 \%$ & 0,85 \\
Hipertensãoarterial sistêmica & $22 \%$ & $18 \%$ & 0,30 \\
Antiinflamatórios & $20 \%$ & $10 \%$ & 0,74 \\
Tabagismo* & $34 \%$ & $21 \%$ & 0,02 \\
Alcoolismo* & $24 \%$ & $26 \%$ & 0,002 \\
Diabetesmellitus* & $8,5 \%$ & $35 \%$ & 0,01 \\
Antec.doençapéptica* & $40 \%$ & $19 \%$ & 0,05 \\
Hemorr.digestivaprévia & $26 \%$ & $17 \%$ & 0,33 \\
Úlceragástrica & $49 \%$ & $13 \%$ & 1 \\
Choqueadmissão* & $13 \%$ & $33 \%$ & $<0,001$ \\
Transfusões* & $54 \%$ & $25 \%$ & $<0,001$ \\
\hline * ${ }^{*} 0$ 05 & & &
\end{tabular}

\begin{tabular}{lccc}
\hline \multicolumn{3}{c}{ Tabela 4-Comparação das médias e desvio padrão das variáveis numéricas entre os operados e não operados } \\
\hline Variável & Operados $\mathbf{N}=\mathbf{2 5}$ & Não operados $\mathbf{N}=\mid \mathbf{7 5}$ & $\mathbf{p}$ \\
Idade(anos) & $57+16$ & $5|+| 8$ & $0,|1| 6$ \\
Hemoglobina(g/dL) & $7,9 \mid+2,3$ & $\mid 0,2+2,8$ & $<0,00 \mid$ \\
Hematócrito & $23,5+7,8$ & $30,6+8,5$ & $<0,00 \mid$ \\
Diâmetroúlcera(mm) & $|7+| 1$ & $8,6+4,6$ & $<0,00 \mid$ \\
Transfusão(Unidadesde & $4,54+2,7$ & $2,42+1,35$ & $<0,00 \mid$ \\
concentradosdehemácias) & & & \\
\hline
\end{tabular}

Tabela 5 - Variáveis associadas à necessidade de tratamento operatório no grupo dos doentes Forrest lb, Ila e llb

\begin{tabular}{lccc}
\hline Variável & Presença na amostra & Operados & $\mathbf{p}$ \\
Sexomasculino & $75 \%$ & $14 \%$ & 0,75 \\
Hipertensãoarterialsistêmica & $22 \%$ & $23 \%$ & 0,21 \\
Antinflamatórios & $18 \%$ & $9 \%$ & 0,74 \\
Tabagismo* & $36 \%$ & $23 \%$ & 0,038 \\
Alcoolismo* & $24 \%$ & $28 \%$ & 0,022 \\
Diabetesmellitus & $11 \%$ & $30 \%$ & 0,14 \\
Antecedentesdedoença péptica* & $39 \%$ & $23 \%$ & 0,002 \\
Hemorragiadigestivaprévia & $24 \%$ & $21 \%$ & 0,30 \\
Úlceragástrica & $47 \%$ & $13 \%$ & 0,96 \\
Choqueàadmissão* & $15 \%$ & $33 \%$ & 0,025 \\
\hline *0 $<0,05$ & & &
\end{tabular}

\begin{tabular}{lccc}
\hline \multicolumn{4}{c}{ Tabela 6-Tratamento endoscópico e necessidade de operação nos doentes com lesões Forrest lb, lla e llb } \\
\hline & Operados & Não operados & Total \\
Tratados & 9 & 88 & 97 \\
Não-tratados & 7 & 12 & 19 \\
Total & 16 & 100 & 116 \\
\hline
\end{tabular}

foramoperados etrêsfaleceram. Foramavaliadas as mesmas variáveis acima (Tabela 5). Choque à admissão, antecedentes dealcoolismo, tabagismo oudoençapépticaforamsignificativamenteassociados à necessidade de tratamento operatório.
Especialmente neste grupo, alguns doentes não receberam tratamento endoscópico. Enquanto sete dos 19 não-tratados foram operados, isto ocorreu em apenas nove dos 97 que receberam alguma forma de tratamento endoscópico $(p=0,005)$ (Tabela 6). Quando avaliamos apenas os doentes com lesões Forrest llb, notamos que $31 \%$ dos que não receberam tratamento endoscópico necessitaram operação, 0 que ocorreu em $3 \%$ dos que receberam hemostasia endoscópica (Tabela 7).

\section{Análise dos doentes submetidos a tratamento endoscópico}

Uma vez que o tratamento endoscópico foi um fator significativo na evolução, optou-se por analisar os fatores preditivos de tratamento operatório especificamente nestes doentes. No total, I 10 foram submetidos a alguma forma de hemostasia endoscópica à admissão. As lesões foram classificadas como Forrest la em cinco doentes $(4,5 \%)$, lb em 19 (I7,3\%), Ila em $46(4$ I ,8\%), Ilb em $32(29,1 \%)$ e llc em oito (7,3\%). Quinze (13,6\%) foram operados.

Antecedentes de tabagismo ou alcoolismo, choque e lesões Forrest la foram os fatores preditivos de tratamento operatório (Tabelas 8). Comparando-se com os identificados para toda a amostra, apenas antecedentes de diabetes mellitus e doença péptica não foram confirmados como fatores associados ao tratamento cirúrgico.

\section{Discussão}

A hemorragia digestiva por úlceras pépticas é uma causa freqüente de internação nos serviços de emergência ${ }^{16}$. $O$ aumento da população de idosos e o uso de antiinflamatórios têm contribuído para este problema. Com o desenvolvimento dos métodos de hemostasia endoscópica, atualmente o tratamento operatório é reservado apenas para uma pequena parcela dos doentes. Contudo, existe ainda controvérsia na condução de alguns casos, justamente aqueles mais graves e com maior chance de complicações e morte ${ }^{17}$.

No período de 28 meses deste estudo, admitimos 200 doentes com úlceras pépticas hemorrágicas. Os critérios de exclusão visaram eliminar fatores não relacionados à doença péptica que alterariam a evolução. Aproximadamente 40 apresentavam antecedentes de doença péptica e um quarto da amostra já tinha internação prévia por hemorragia digestiva alta. Provavelmente tratava-se de doença crônica, com falha do tratamento ambulatorial.

O tratamento endoscópico foi realizado em $55 \%$ dos doentes, pois apenas uma parcela 


\begin{tabular}{lccc}
\hline \multicolumn{4}{c}{ Tabela 7 } \\
\hline & Tratamento endoscópico e enecessidade de operação nos doentes com lesóes Forrest llb \\
\hline Tratados & Operados & Não operados & Total \\
Não-tratados & 1 & 31 & 32 \\
Total & 5 & 11 & 16 \\
\hline
\end{tabular}

Tabela 8 - Fatores preditivos de tratamento operatório nos doentes submetidos a hemostasia endoscópica à admissão $(n=110)$

\begin{tabular}{lccc}
\hline Varável & Presença na amostra & Operados & $\mathrm{p}$ \\
Hipertensãoarterialsistêmica & $20 \%$ & $18 \%$ & 0,72 \\
Antiinflamatórios & $15 \%$ & $17 \%$ & 0,88 \\
Tabagismo* & $36 \%$ & $25 \%$ & 0,019 \\
Alcoolismo* & $23 \%$ & $30 \%$ & 0,01 \\
Diabetesmellitus & $6 \%$ & $28 \%$ & 0,53 \\
Antecedentesdedoençapéptica & $37 \%$ & $17 \%$ & 0,60 \\
Úlceragástrica & $54 \%$ & $12 \%$ & 0,76 \\
Choqueàadmissão* & $15 \%$ & $35 \%$ & 0,014 \\
Forrestla* & $4 \%$ & $100 \%$ & $<0,001$ \\
\hline${ }^{*} p<0,05$ & & &
\end{tabular}

das lesões apresentava sinais de sangramento ativo na endoscopia digestiva da admissão. Contudo, na análise dos doentes Forrest lb, lla e llb, técnicas de hemostasia endoscópica foram empregadas em $83 \%$. O tratamento operatório foi necessário em apenas $12,5 \% \mathrm{da}$ amostra, ratificando a efetividade dos métodos endoscópicos de hemostasia, mesmo na presença de sangramento ativo "difuso", coto vascular ou coágulo aderido à lesão.

Cerca de $54 \%$ da amostra recebeu transfusões de concentrados de hemácias. A princípio poder-se-ia sugerir que houve atraso na indicação cirúrgica, determinando maior sangramento. Contudo, $44 \%$ dos doentes foram admitidos com concentração sérica de hemoglobina inferior a $10 \mathrm{~g} / \mathrm{dL}$, o que certamente foi o fator principal na indicação das transfusões.

Na recidiva hemorrágica, seis doentes do total repetiram o exame endoscópico, sendo 0 restante submetido diretamente ao tratamento operatório. Alguns autores relatam uma segunda tentativa de controle endoscópico do sangramento em até $76 \%$ dos $\operatorname{casos}^{16}$, contudo, em nossa amostra, a escolha pela operação baseou-se principalmente na cronicidade da doença péptica associada a sangramentos digestivos repetidos.

Não há dados que demonstrem claramente que uma segunda endoscopia para tratamento destes doentes seria a melhor opção ${ }^{18}$. Embora alguns autores relatem bons resultados com uma segunda tentativa de hemostasia endoscópica, a sua realização poderia representar um fator a mais retardando o tratamento operatório adequado, 0 que favoreceria um pior prognóstico ${ }^{8}$. Devese ressaltar que a injeção de adrenalina ou álcool absoluto na mucosa gastroduodenal não é indene, e pode interferir no resultado final do tratamento do coto duodenal ${ }^{17}$. Com a evolução dos métodos mecânicos de hemostasia endoscópica, eventualmente este cenário pode ser modificado ${ }^{19}$. Outra opção seria a realização de uma segunda endoscopia digestiva "controle" (Second look endoscopy), na tentativa de diagnosticar precocemente o ressangramento e melhorar a eficácia do tratamento endoscópico ${ }^{5,20}$.

Acreditamos que na recidiva hemorrágica a conduta é determinada pela análise de vários fatores relacionados a cada doente. Nos jovens, sem antecedentes de doença péptica, que ingeriram algum antiinflamatório não esteróide e com lesões duodenais pequenas, uma segunda tentativa de hemostasia endoscópica deve ser preconizada. No outro extremo encontramos os idosos, com doença péptica crônica e lesões gástricas de grandes dimensões, nos quais a operação é a melhor forma de tratamento. Contudo, a maioria dos casos está entre estes dois exemplos, e a indicação operatória nem sempre é fácil.

Quando indicada, a operação quase sempre inclui a ressecção gástrica devido às características das lesões ${ }^{17}$. Em nossa amostra, $92 \%$ dos operados foram submetidos à gastrectomia parcial com reconstrução à Billroth II. Freqüentemente tratava-se de úlceras terebrantes para pâncreas ou outros órgãos, de dimensões consideráveis, e que não permitiam outra forma de tratamento.

A letalidade geral da amostra foi $3 \%$. Entretanto, cerca de $24 \%$ dos doentes operados faleceram, e complicações relacionadas à operação foram responsáveis por um terço dos óbitos. Em doentes submetidos gastrectomias realizadas na urgência, So et al. associam a mortalidade à idade avançada, choque e dosagem de hemoglobina sérica menor que $10 \mathrm{~g} / \mathrm{dL}^{10}$. Dos doentes operados, $40 \%$ tinham idade avançada, $36 \%$ foram admitidos em choque e $80 \%$ tinham dosagem sérica de hemoglobina inferior a $10 \mathrm{~g} / \mathrm{dL}$. Isto se relaciona com a alta letalidade destes casos. Aparentemente, as complicações operatórias são pouco freqüentes, mas quando ocorrem são graves.

Questiona-se se algo diferente poderia ter sido feito para mudar a evolução destes doentes. Os bloqueadores de bomba de prótons são mais efetivos que os bloqueadores $\mathrm{H} 2$ na prevenção da recidiva hemorrágica ${ }^{21}, \mathrm{e}, \mathrm{em}$ nosso estudo, empregou-se ranitidine. Tal efeito seria secundário ao controle do $\mathrm{pH}$ gástrico, fornecendo melhores condições de coagulação no sítio hemorrágico ${ }^{22,23}$. $\mathrm{O}$ emprego destas drogas pode encontrar limitações pelo custo envolvido, e nem sempre está disponível em hospitais públicos.

Outro fator importante a ser analisado éo momento da operação. Apesar de não haver estudo prospectivo randomizado a respeito, acredita-se que a operação precoce, antes da descompensação clínica, seja a melhor opção para os doentes idosos e de maior risco" ". Isto tem sentido se observarmos os fatores prognósticos nos doentes submetidos à ressecção gástrica na urgência, que são justamente a instabilidade hemodinâmica, anemia aguda e idade avançada ${ }^{10}$.

Assim sendo, considera-se de grande valor a identificação precoce dos doentes com maior chance de necessitar tratamento operatório. 
Isto poderia auxiliar o endoscopista na escolha do método hemostático, indicar uma revisão endoscópica ou mesmo o tratamento cirúrgico antes da deterioração clínica. Em nosso estudo, os fatores associados à necessidade de tratamento operatório foram presença de choque à admissão, antecedentes de alcoolismo, tabagismo ou doença péptica crônica, assim como úlceras Forrest la. Estes resultados são semelhantes aos encontrados por outros autores 8,24 .

Thomopoulos et al., em 200I, associaram a falha do tratamento endoscópico à presença de choque à admissão, úlceras com sangramento em jato ou localizadas na parede posterior do duodeno ${ }^{24}$. Além do choque à admissão e sangramento ativo em jato, Chung et al. encontraram associação de falha da hemostasia endoscópica com úlceras com diâmetro maior que dois centímetros ou localizadas em estômago ${ }^{8}$. Tanto nestes estudos como na nossa casuística, não houve relação da faixa etária com a maior necessidade de tratamento operatório, o que não é um consenso. Branicki et al., em 1990, relataram que em doentes com mais de 60 anos de idade, a recidiva de sangramento após o tratamento endoscópico era mais freqüente ${ }^{25}$.

Dados contraditórios foram encontrados a respeito da localização da úlcera. Historicamente, úlceras localizadas em parede posterior de duodeno ou pequena curvatura gástrica são associadas a maior necessidade de tratamento operatório ${ }^{14}$. Não encontramos tal relação em nossa amostra. Provavelmente, existem outros fatores mais importantes para o ressangramento, como o diâmetro da lesão e a classificação de Forrest.

Em nosso estudo, os doentes com lesões Forrest llb que não receberam alguma forma de hemostasia endoscópica na admissão apresentaram uma maior freqüência de ressangramento e necessidade de tratamento cirúrgico. Isto é relevante, uma vez que alguns autores questionam a necessidade de tratamento endoscópico nesses casos. Em uma pesquisa nacional holandesa, Van Leerdam et al. relatam que, dos médicos questionados, apenas $47 \%$ realizam terapia endoscópica para estas lesões ${ }^{16}$. Os dados do nosso estudo sugerem que a hemostasia endoscópica deva ser realizada obrigatoriamente, na tentativa de diminuir a chance de ressangramento.
Há uma forte relação entre descompensação clínica, falha da hemostasia endoscópica, necessidade de tratamento operatório e letalidade pós-operatória. A instabilidade clínica pode representar tanto causa como conseqüência do ressangramento, o que gera um ciclo vicioso. Conseqüentemente, os doentes com maior risco cirúrgico são os mais propensos a necessitar de operação. Quando são operados, normalmente apresentamúlceras pépticas de difícil tratamento operatório, representado pela dificuldade de síntese do duodeno. Logo, são também mais suscetíveis a complicações pós-operatórias, tanto pelas doenças associadas como pela dificuldade técnica intra-operatória. Oresultado observado é a alta letalidade dos doentes operados, comparada à da amostra em geral.

Enfim, ainda há vários pontos em discussão a respeito do tratamento das úlceras pépticas hemorrágicas. A despeito do progresso no tratamento endoscópico, a operação ainda é necessária em alguns casos, e traz uma mortalidade considerável. A avaliação dos fatores associados ao tratamento operatório pode diminuir o tempo atéa hemostasia definitivae, desta forma, possivelmente melhorar o prognóstico destes doentes.

\section{SUMMARY}

Predictors of operative treatment in PATIENTS SUSTAINING BLEEDING PEPTIC ULCERS

OBJECTIVE. To analyze the predictors of operative treatment in patients sustaining bleeding peptic ulcers.

METHODS. Retrospective analysis of the patients admitted owing to bleeding peptic ulcers from 1998 to 200I. Vital signs upon admission, associated diseases, endoscopy data, evolution and definitive care were evaluated. Patients who underwent surgery were compared with the others in order to identify the predictors of the operative treatment. Student's $t$ and chi squared test were employed for statistical analysis, considering $p<0,05$ as significant.

RESULTS. Two hundred patients were included in the study group. The age ranged between 17 and 97 years (mean $52 \pm 18$ ), and 153 patients $(76.5 \%)$ were male. Shock upon admission was present in 27 (13.5\%). The endoscopic exam performed at admission showed that 101 patients sustained ulcers in the duodenum and
99 in the stomach, which were classified as Forrest la in 5 (2.5\%), Ib in 20 (10\%), Ila in 48 (24\%) and llb in 48 (24\%). Twenty five (I2.5\%) patients needed operation, and $23(92 \%)$ were submitted to gastrectomy with Billroth's II reconstruction. The operative treatment were more frequently performed in patients sustaining chronic alcoholic intake $(p=0.002)$, cigarette smoking $(p=0.02)$, diabetes mellitus $(p=0.01)$, chronic peptic disease $(p=0.05)$, shock upon admission $(p<0.00 I)$ and spurting activity at the endoscopy $(p<0.001)$. Forrest llb lesions which did not receive any form of endoscopic treatment at admission were more likely to rebleed $(p=0.012)$.

Conclusions. The predictors of operative treatment were chronic alcoholic intake, smoke addiction, diabetes mellitus, chronic peptic disease, shock upon admission and Forrest la ulcers. [Rev Assoc Med Bras 2002; 48(4): 297-302]

KeYwords: Peptic ulcer. Peptic ulcer hemorrhage - therapy and surgery.

\section{REFERÊNCIAS}

I. Spirt M. Upper gastrointestinal bleeding: peptic ulcer disease. In: Spirt M. Acute care of the abdomen. Baltimore: Willians \& Wilkins; 1998. p.23-48.

2. Gabriel SE, Jaakkimainen L, Bombardier C. Risk of serious gastrointestinal complications related to use of nonsteroidal anti-inflammatory drugs: a meta-analysis. Ann Intern Med 1991; I I 5:787-96.

3. Allison MC, Howatson AG, Torrence CJ, Lee FD, Russel RI. Gastrointestinal damage associated with the use of nonsteroidal antiinflammatory drugs. N Engl J Med 1992; 327:749-54.

4. Laine L, Cohen H, Brodhead J. Prospective evaluation of immediate versus delayed refeeding and prognostic value of endoscopy in patients with upper gastrointestinal hemorrhage. Gastroenterology 1992; 102:314-6.

5. Trap R, Skarbye M, Rosemberg. Planned second look endoscopy in patients with bleeding duodenal or gastric ulcer. Dan Med Bull 2000; 47:200-3.

6. Church NI, Palmer KR. Relevance of the Rockall score in patients undergoing endoscopic therapy for peptic ulcer haemorrhage. Eur J Gastroenterol Hepatol 200 I ; I : I | 49-52.

7. Chung YF, Wong WK, Soo KC. Diagnostic failures for acute upper gastrointestinal haemorrhage. Br J Surg 2000; 87:6I4-7.

8. Chung IK, Kim EJ, Lee MS, Kim HS, Park SH, Lee $\mathrm{MH}$, et al. Endoscopic factors predisposing to rebleeding following endoscopic hemostasis in bleeding peptic ulcers. Endoscopy 200 I; 33:969-75.

9. Gama-Rodrigues J, Bresciani CJC. Úlcera 
péptica hemorrágica. In: Rasslan S. Afecções cirúrgicas de urgência. São Paulo: Robe Editorial; 1995. p. 195-208.

I0. So JB, Yam A, Cheah WK, Kum CK, Goh PM. Risk factors related to operative mortality and morbidity in patients undergoing emergency gastrectomy. Br J Surg 2000; 87: I 702-7.

II. Lau JY, Chung SC. Surgery in the acute management of bleeding peptic ulcer. Baillieres Best Pract Res Clin Gastroenterol 2000; 14:505-18.

12. Millat B, Fingerhut A, Borie F. Surgical treatment of complicated duodenal ulcers. World J Surg 2000; 24:299-306.

13. Freeman ML.Value of stigmatain decision-making in gastrointestinal haemorrhage. Baillieres Best Pract Res Clin Gastroenterol 2000; 14:4 I I-25.

14. LinHJ, perng CL, LeeFY, LeeCH, LeeSD. Clinical courses and predictors for rebleeding in patients with pepticulcers and non-bleedingvisiblevessels: a prospective study. Gut 1994; 35: 1389-93.

I5. Forest JA, Finlayson ND, Shearman DJ. Endoscopy in gastrointestinal bleeding. Lancet 1974; 17:394-7.

16. Van Leerdam ME, Rauws EA, Geraedts AA,
Tytgat GN. Management in peptic ulcer hemorrhage: a Dutch national inquiry. Endoscopy 2000; 32: 935-42.

17. Rasslan S, Gama-Rodrigues J. Tratamento da úlcera péptica sangrante: análise crítica do tratamento operatório. In: Wazen da Rocha, J. Hemorragia digestiva alta. Rio de Janeiro: Atheneu; 2002. (no prelo).

18. Ohmann C, Imhof M, Roher HD. Trends in peptic ulcer bleeding and surgical treatment. World J Surg 2000; 24:284-93.

19. Hepworth CC, Swain CP. Mechanical endoscopic methods of haemostasis for bleeding peptic ulcers: a review. Baillieres Best Pract Res Clin Gastroenterol 2000; 14:467-76.

20. Asaki S. Efficacy of endoscopic pure ethanol injection method for gastrointestinal ulcer bleeding. World J Surg 2000; 24: 294-8.

2I. Javid G, Masoodi I, Zagar SA, Khan BA, Yatoo $\mathrm{GN}$, Shah AH, et al. Omeprazole as adjuvant therapy to edoscopic combination ijection sclerotherapy for treating bleeding peptic ulcer.Am J Med 200 I; I I:280-4.

22. LiY, Sha W, Nie Y, Wu H, She Q, Dai S, et al.
Effect of intragastric $\mathrm{pH}$ on control of peptic ulcer bleeding. J Gastroenterol Hepatol 2000; 15:148-54.

23. Van Leerdam ME, Rauws EA. The role of acid suppressants in upper gastrointestinal ulcer bleeding. Best Pract Res Clin Gastroenterol 200I; 15: 463-75.

24. Thomopoulos KC, Mitropoulos JA, Katsakoulis EC, Vagianos CE, Mimidis KP, Hatziargiriou $\mathrm{MN}$, et al. Factors associated with failure of endoscopic injection haemostasis in bleeding peptic ulcers. Scand J Gastroenterol 200I; 36:664-8

25. Branicki FJ, BoeyJ, Pok PJ, Pritchett CJ, Fan ST, Lai EC. Bleeding duodenal ulcer. A prospective evaluation of risk factors for rebleeding and death. Am Surg 1990; 21 I:4 I I-8.

Artigo recebido: 19/03/2002 Aceito para publicação: 06/05/2002 